International Journal of Pure and Applied Mathematics

Volume 106 No. 2 2016, 473-479

ISSN: 1311-8080 (printed version); ISSN: 1314-3395 (on-line version)

url: http://www.ijpam.eu

doi: 10.12732/ijpam.v106i2.11

\title{
DISCRETE HARDY-TYPE INEQUALITY WITH KERNEL
}

\author{
Suket Kumar \\ Department of Mathematics \\ NIT Hamirpur \\ Himachal Pradesh, 177005, INDIA
}

\begin{abstract}
Necessary and sufficient conditions are given for the validity of discrete Hardy's inequality for the sum of two discrete Hardy-type operators with not necessary non-negative coefficients.
\end{abstract}

AMS Subject Classification: 26D10, 26D15

Key Words: discrete hardy inequality, weighted sequence space and its norm, duality

\section{Introduction}

Discrete Hardy inequality has been characterized for the discrete Hardy operator in $[2,3,9]$. Sufficient condition is available in [1] for the validity of the discrete Hardy inequality, for the discrete Hardy-type operator with kernel $k=\left\{k_{m, n}\right\}$; $m, n \in Z_{+}$defined on $D=\left\{(m, n) \in Z_{+} \times Z_{+}: n \leq m\right\}, k_{m, n}$ is non-increasing in $m$ and non-decreasing in $n$. For the discrete Hardy-type operator with general kernel, sufficient conditions for the validity of discrete Hardy inequality is available in [8] which is both necessary and sufficient when the kernel is of product type.

In this note, we consider a different discrete operator

$$
T: \ell^{p}\left(v_{n}\right) \rightarrow \ell^{q}\left(u_{n}\right)
$$

defined as

$$
T\left(a_{n}\right)=\phi_{1, m} \sum_{n=1}^{m} \psi_{1, n} k_{m, n} a_{n}+\phi_{2, m} \sum_{n=m}^{\infty} \psi_{2, n} k_{n, m} a_{n}
$$

where the kernel involve is discrete Oinarov kernel (not considered in earlier

Received: October 3, 2015

Published: February 15, 2016
(C) 2016 Academic Publications, Ltd.

url: www.acadpubl.eu 
literature) and characterize Hardy inequality. Here $\left\{\phi_{i, n}\right\},\left\{\psi_{i, n}\right\} ; i=1,2$ and $\left\{a_{n}\right\}$ are sequences of real numbers not necessarily non-negative. Therefore $T$ is not necessarily non-negative. Hardy inequality for the operator $T$ gives better best constant for the Hardy inequality than the same for the single discrete Hardy-type operator. Our study is motivated by this.

Kernel $k=\left\{k_{m, n}\right\} ; m, n \in Z_{+}$is called discrete Oinarov kernel if (a) $k_{m, n} \geq$ 0 for $0<n<m$; (b) $k_{m, n}$ is increasing in $m$ and decreasing in $n$, (c) $k_{m, n} \approx$ $k_{m, p}+k_{p \cdot n}$ for $0<n<p<m$.

Throughout the paper, $n, m, \alpha, \beta$ are positive integers $\left(Z_{+}\right) ; t$ is fixed positive integer; $p, q$ are real numbers; $p^{\prime}=p /(p-1)$ is conjugate to $p$ and the same is true for $q \cdot \ell^{p}\left(v_{n}\right)$ denotes weighted sequence space. $\left\{u_{n}\right\},\left\{v_{n}\right\} ; n \in Z_{+}$are weight sequences.

For complete description of Hardy inequality, we use to refer [5] and monographs $[6,7]$.

\section{Preliminaries}

In the following result, we state the discrete case of [7, Theorem 2.10]:

Proposition 2.1. Suppose $1<p \leq q<\infty,\left\{u_{n}\right\},\left\{v_{n}\right\}$ are weight sequences and $k=\left\{k_{m, n}\right\} ; m, n \in Z_{+}$is discrete Oinarov kernel. The inequality

$$
\left(\sum_{m=1}^{\infty}\left|\sum_{n=1}^{m} k_{m, n} a_{n}\right|^{q} u_{m}\right)^{\frac{1}{q}} \leq C\left(\sum_{m=1}^{\infty}\left|a_{m}\right|^{p} v_{m}\right)^{\frac{1}{p}}
$$

holds for all non-negative sequence $\left\{a_{n}\right\} \in \ell^{p}\left(v_{n}\right)$ and a suitable positive constant $C$ if and only if $\max (A, B)<\infty$, where

$$
\begin{aligned}
& A=\sup _{m \in Z_{+}}\left(\sum_{n=m}^{\infty} k_{n, m}^{q} u_{n}\right)^{\frac{1}{q}}\left(\sum_{n=1}^{m} v_{n}^{1-p^{\prime}}\right)^{\frac{1}{p^{\prime}}}, \\
& B=\sup _{m \in Z_{+}}\left(\sum_{n=m}^{\infty} u_{n}\right)^{\frac{1}{q}}\left(\sum_{n=1}^{m} k_{m, n}^{p^{\prime}} v_{n}^{1-p^{\prime}}\right)^{\frac{1}{p^{\prime}}} .
\end{aligned}
$$

Proof. The proof of Proposition 2.1 is similar to continuous case. Continuous case was first proved in [4]. We omit the detail.

By using duality argument and applying suitable substitutions in Proposition (2.1), we can prove the following. 
Proposition 2.2. Suppose $1<p \leq q<\infty,\left\{u_{n}\right\},\left\{v_{n}\right\}$ are weight sequences and $k=\left\{k_{n, m}\right\} ; n, m \in Z_{+}$is discrete Oinarov kernel. The inequality

$$
\left(\sum_{m=1}^{\infty}\left|\sum_{n=m}^{\infty} k_{n, m} a_{n}\right|^{q} u_{m}\right)^{\frac{1}{q}} \leq C\left(\sum_{m=1}^{\infty}\left|a_{m}\right|^{p} v_{m}\right)^{\frac{1}{p}}
$$

holds for all non-negative sequence $\left\{a_{n}\right\} \in \ell^{p}\left(v_{n}\right)$ and a suitable positive constant $C$ if and only if $\max (D, E)<\infty$ where

$$
\begin{aligned}
& D=\sup _{m \in Z_{+}}\left(\sum_{n=1}^{m} k_{m, n}^{q} u_{n}\right)^{\frac{1}{q}}\left(\sum_{n=m}^{\infty} v_{n}^{1-p^{\prime}}\right)^{\frac{1}{p^{\prime}}}, \\
& E=\sup _{m \in Z_{+}}\left(\sum_{n=1}^{m} u_{n}\right)^{\frac{1}{q}}\left(\sum_{n=m}^{\infty} k_{n, m}^{p^{\prime}} v_{n}^{1-p^{\prime}}\right)^{\frac{1}{p^{\prime}}} .
\end{aligned}
$$

\section{Main Result}

Now, we prove our main result.

Theorem 3.1. Suppose $1<p \leq q<\infty,\left\{\phi_{i, n}\right\},\left\{\psi_{i, n}\right\} ; i=1,2$, are sequences of real numbers not necessarily non-negative, $\left\{u_{n}\right\},\left\{v_{n}\right\}$ are weight sequences and $k=\left\{k_{m, n}\right\} ; m, n \in Z_{+}$is discrete Oinarov kernel. The inequality

$$
\begin{aligned}
\left(\sum_{m=1}^{\infty}\left|\phi_{1, m} \sum_{n=1}^{m} k_{m, n} \psi_{1, n} a_{n}+\phi_{2, m} \sum_{n=m}^{\infty} k_{n, m} \psi_{2, n} a_{n}\right|^{q} u_{m}\right)^{\frac{1}{q}} & \leq C\left(\sum_{m=1}^{\infty}\left|a_{m}\right|^{p} v_{m}\right)^{\frac{1}{p}}
\end{aligned}
$$

holds for all sequence $\left\{a_{n}\right\} \in \ell^{p}\left(v_{n}\right)$ not necessarily non-negative and a suitable positive constant $C$ if and only if $\max (F, G, H, K)<\infty$, where

$$
\begin{aligned}
& F=\sup _{m \in Z_{+}}\left(\sum_{n=m}^{\infty} k_{n, m}^{q}\left|\phi_{1, n} u_{n}^{\frac{1}{q}}\right|^{q}\right)^{1 / q}\left(\sum_{n=1}^{m}\left|\psi_{1, n} v_{n}^{-\frac{1}{p}}\right|^{p^{\prime}}\right)^{1 / p^{\prime}}, \\
& G=\sup _{m \in Z_{+}}\left(\sum_{n=m}^{\infty}\left|\phi_{1, n} u_{n}^{\frac{1}{q}}\right|^{q}\right)^{\frac{1}{q}}\left(\sum_{n=1}^{m} k_{m, n}^{p^{\prime}}\left|\psi_{1, n} v_{n}^{-\frac{1}{p}}\right|^{p^{\prime}}\right)^{\frac{1}{p^{\prime}}},
\end{aligned}
$$




$$
\begin{aligned}
& H=\sup _{m \in Z_{+}}\left(\sum_{n=1}^{m} k_{m, n}^{q}\left|\phi_{2, n} u_{n}^{\frac{1}{q}}\right|^{q}\right)^{\frac{1}{q}}\left(\sum_{n=m}^{\infty} \mid \psi_{2, n} v_{n}^{\left.-\left.\frac{1}{p}\right|^{p^{\prime}}\right)^{\frac{1}{p^{\prime}}}},\right. \\
& K=\sup _{m \in Z_{+}}\left(\sum_{n=1}^{m}\left|\phi_{2, n} u_{n}^{\frac{1}{q}}\right|^{q}\right)^{\frac{1}{q}}\left(\sum_{n=m}^{\infty} k_{n, m}^{p^{\prime}} \mid \psi_{2, n} v_{n}^{-\left.\frac{1}{p}\right|^{p^{\prime}}}\right)^{\frac{1}{p^{\prime}}} .
\end{aligned}
$$

Proof. Sufficiency. By an application of Proposition (2.1) we find that $\max (F, G)<\infty$ holds implies the inequality

$$
\left(\sum_{m=1}^{\infty}\left|\phi_{1, m} \sum_{n=1}^{m} k_{m, n} \psi_{1, n} a_{n}\right|^{q} u_{m}\right)^{\frac{1}{q}} \leq C\left(\sum_{m=1}^{\infty}\left|a_{m}\right|^{p} v_{m}\right)^{\frac{1}{p}}
$$

holds for a positive constant C.

Analogously, making similar arguments and using Proposition (2.2), we can prove that $\max (H, K)<\infty$ holds implies the inequality

$$
\left(\sum_{m=1}^{\infty}\left|\phi_{2, m} \sum_{n=m}^{\infty} k_{n, m} \psi_{2, n} a_{n}\right|^{q} u_{m}\right)^{\frac{1}{q}} \leq C\left(\sum_{m=1}^{\infty}\left|a_{m}\right|^{p} v_{m}\right)^{\frac{1}{p}}
$$

holds for a positive constant C.

Sufficiency now follows from the inequality

$$
\begin{aligned}
& \left(\sum_{m=1}^{\infty}\left|\phi_{1, m} \sum_{n=1}^{m} k_{m, n} \psi_{1, n} a_{n}+\phi_{2, m} \sum_{n=m}^{\infty} k_{n, m} \psi_{2, n} a_{n}\right|^{q} u_{m}\right)^{\frac{1}{q}} \\
& \leq\left(\sum_{m=1}^{\infty}\left|\phi_{1, m} \sum_{n=1}^{m} k_{m, n} \psi_{1, n} a_{n}\right|^{q} u_{m}\right)^{\frac{1}{q}}+\left(\sum_{m=1}^{\infty}\left|\phi_{2, m} \sum_{n=m}^{\infty} k_{n, m} \psi_{2, n} a_{n}\right|^{q} u_{m}\right)^{\frac{1}{q}} .
\end{aligned}
$$

Necessity. For $\varepsilon>0, n<t<m$ and $1 \leq \alpha<\beta<\infty$ we define modified weight sequence $\left\{v_{n_{\varepsilon}}\right\}$ where

$$
v_{n_{\varepsilon}}=\max \left\{v_{n}, k_{t, n}^{p}\left|\psi_{1, n}\right|^{p} \varepsilon\right\}
$$

and sequence $\left\{a_{t, n}\right\}$ where

$$
a_{t, n}= \begin{cases}\left\{k_{t, n} \mid \psi_{1, n} v_{n_{\varepsilon}}^{-1 / p}\right\}^{p^{\prime}-1} \operatorname{sgn}\left\{\psi_{1, n} v_{n_{\varepsilon}}^{-1 / p}\right\} v_{n_{\varepsilon}}^{-1 / p}, & \alpha<n<\beta \\ 0, & \text { otherwise }\end{cases}
$$


For the above choice, the L.H.S. of (3.1) becomes

$$
\begin{gathered}
\left(\sum_{m=1}^{\infty}\left|\phi_{1, m} \sum_{n=1}^{m} k_{m, n} \psi_{1, n} a_{t, n}+\phi_{2, m} \sum_{n=m}^{\infty} k_{n, m} \psi_{2, n} a_{t, n}\right|^{q} u_{m}\right)^{\frac{1}{q}} \\
\geq\left(\sum_{m=\beta}^{\infty}\left|\phi_{1, m} \sum_{n=1}^{m} k_{m, n} \psi_{1, n} a_{t, n}+\phi_{2, m} \sum_{n=m}^{\infty} k_{n, m} \psi_{2, n} a_{t, n}\right|^{q} u_{m}\right)^{\frac{1}{q}} \\
=\left(\sum_{m=\beta}^{\infty}\left|\phi_{1, m} \sum_{n=1}^{m} k_{m, n} \psi_{1, n} a_{t, n}\right|^{q} u_{m} \mid \begin{array}{l}
1 / q \\
\geq
\end{array}\right. \\
\left.\left(\sum_{m=\beta}^{\infty} u_{m}\left|\phi_{1, m}\right|^{q}\left(\sum_{n=\alpha}^{\beta} k_{m, n} k_{t, n}^{p^{\prime}-1}\left|\psi_{1, n} v_{n_{\varepsilon}}^{-1 / p}\right|^{p^{\prime}}\right)^{q}\right)^{1 / q}=0 \text { for } n \geq \beta\right) \\
\quad\left(\operatorname{Since}\left(\psi_{1, n} v_{n_{\varepsilon}}^{-1 / p}\right) \operatorname{sgn}\left\{\psi_{1, n} v_{n_{\varepsilon}}^{-\frac{1}{p}}=\left|\psi_{1, n} v_{n_{\varepsilon}}^{-\frac{1}{p}}\right|\right)\right. \\
\quad\left(\sum_{m=\beta}^{\infty} u_{m}\left|\phi_{1, m}\right|^{q}\left(\sum_{n=\alpha}^{\beta} k_{t, n}^{p^{\prime}}\left|\psi_{1, n} v_{n_{\varepsilon}}^{-1 / p}\right|^{p^{\prime}}\right)^{q} \mid\right.
\end{gathered}
$$

$\left(k_{m, n}\right.$ is Oinarov kernal implies $k_{m, n}>k_{t, n}$ for $m>t>n$ )

$$
=\left(\sum_{m=\beta}^{\infty} u_{m}\left|\phi_{1, m}\right|^{q}\right)^{\frac{1}{q}}\left(\sum_{n=\alpha}^{\beta} k_{t, n}^{p^{\prime}}\left|\psi_{1, n} v_{n_{\varepsilon}}^{-\frac{1}{p}}\right|^{p^{\prime}}\right),
$$

while the R.H.S. of (3.1) can be estimated as

$$
\begin{aligned}
C\left(\sum_{n=1}^{\infty}\left|a_{t, n}\right|^{p} v_{n}\right)^{\frac{1}{p}} & \leq C\left(\sum_{n=1}^{\infty}\left|a_{t, n}\right|^{p} v_{n_{\varepsilon}}\right)^{\frac{1}{p}} \\
& =C\left(\sum_{n=\alpha}^{\beta} k_{t, n}^{p^{\prime}}\left|\psi_{1, n} v_{n_{\varepsilon}}^{-\frac{1}{p}}\right|^{p^{\prime}}\right)^{1 / p^{\prime}} \\
& \leq C \varepsilon^{1-p^{\prime}}(\beta-\alpha)^{1 / p}<\infty
\end{aligned}
$$

Consequently the inequality (3.1) yields

$$
\left(\left.\sum_{m=\beta}^{\infty} u_{m}\left|\phi_{1, m}\right|^{q}\right|^{1 / q}\left(\sum_{n=\alpha}^{\beta} k_{t, n}^{p^{\prime}}\left|\psi_{1, n} v_{n_{\varepsilon}}^{-\frac{1}{p}}\right|^{p^{\prime}}\right)^{1 / p^{\prime}} \leq C<\infty,\right.
$$


which holds for a positive constant $C$ independent of $\alpha$ and $\varepsilon$. For $\alpha \rightarrow 1$ and $\varepsilon \rightarrow 0$ (via a subsequence), we get $v_{n_{\varepsilon}} \rightarrow v_{n}$ and consequently taking supremum over all integer $\beta \geq 1$, we find that $G$ is finite.

Repeating the above process in the inequality (3.1) with $v_{n_{\varepsilon}}$ and $a_{t, n}$ replaced, respectively, by

$$
\tilde{v}_{n_{\varepsilon}}=\max \left\{k_{n, t}^{p}\left|\psi_{2, n}\right|^{p} \varepsilon, v_{n}\right\}
$$

and

$$
\tilde{a}_{n, t}= \begin{cases}\left\{k_{n, t}\left|\psi_{2, n} \tilde{v}_{n_{\varepsilon}}^{-1 / p}\right|\right\}^{p^{\prime}-1} \operatorname{sgn}\left\{\psi_{2, n} \tilde{v}_{n_{\varepsilon}}^{-1 / p}\right\} \tilde{v}_{n_{\varepsilon}}^{-1 / p}, & \alpha<n<\beta, \\ 0, & \text { otherwise },\end{cases}
$$

with $m<t<n$ and $1 \leq \alpha<\beta<\infty$, we find $K<\infty$.

Conjugate operator to $T$ denoted by $T^{*}$ is defined as

$$
T^{*}\left(a_{n}\right)=\psi_{1, m} \sum_{n=m}^{\infty} k_{n, m} \phi_{1, n} a_{n}+\psi_{2, m} \sum_{n=1}^{m} k_{m, n} \phi_{2, n} a_{n} .
$$

By using duality argument it may be shown that the inequality (3.1) and

$$
\left\|T^{*}\left(a_{n}\right)\right\|_{p^{\prime}, v_{n}^{1-p^{\prime}}} \leq C\left\|a_{n}\right\|_{q^{\prime}, u_{n}^{1-q^{\prime}}}
$$

are equivalent, where norm used is that of weighted sequence space. For a new weight sequence $\left\{u_{n_{\varepsilon}}\right\}$ where

$$
u_{n_{\varepsilon}}=\min \left\{u_{n}, k_{t, n}^{-q}\left|\phi_{2, n}\right|^{-q} \varepsilon^{-1}\right\}
$$

we find that $\left\|a_{n}\right\|_{q^{\prime}, u_{n}^{1-q^{\prime}}} \leq\left\|a_{n}\right\|_{q^{\prime}, u_{n_{\varepsilon}}^{1-q^{\prime}}}$ holds and therefore, the inequality (3.2) yields

$$
\left\|T^{*}\left(a_{n}\right)\right\|_{p^{\prime}, v_{n}^{1-p^{\prime}}} \leq C\left\|a_{n}\right\|_{q^{\prime}, u_{n_{\varepsilon}}^{1-q^{\prime}}} .
$$

For $1 \leq \alpha<\beta<\infty$ and $n<t<m$, define a sequence $\left\{g_{t, n}\right\}$ where

$$
g_{t, n}= \begin{cases}\left\{k_{t, n}\left|\phi_{2, n} u_{n_{\varepsilon}}^{1 / q}\right|\right\}^{q-1} \operatorname{sgn}\left\{\phi_{2, n} u_{n_{\varepsilon}}^{1 / q}\right\} u_{n_{\varepsilon}}^{1 / q}, & \alpha<n<\beta, \\ 0, & \text { otherwise. }\end{cases}
$$

Substituting $a_{n}=g_{t, n}$ in the inequality (3.3) and using similar argument, we find $H<\infty$. Repeating the above process in the inequality (3.3) with $u_{n_{\varepsilon}}$ and $g_{t, n}$ replaced, respectively, by

$$
\tilde{u}_{n_{\varepsilon}}=\min \left\{u_{n}, k_{n, t}^{-q}\left|\phi_{1, n}\right|^{-q} \varepsilon^{-1}\right\}
$$


and

$$
\tilde{g}_{t, n}= \begin{cases}\left\{k_{n, t}\left|\phi_{1, n} \tilde{u}_{n_{\varepsilon}}^{1 / q}\right|\right\}^{q-1} \operatorname{sgn}\left\{\phi_{1, n} \tilde{u}_{n_{\varepsilon}}^{1 / q}\right\} \tilde{u}_{n_{\varepsilon}}^{1 / q}, & \alpha<n<\beta \\ 0, & \text { otherwise }\end{cases}
$$

where $m<t<n$ and $1 \leq \alpha<\beta<\infty$, we find $F<\infty$. The necessity is now proved.

\section{References}

[1] K.F. Andersen and H.P. Heinig, Weighted norm inequalities for certain integral operators, SIAM J. Math. Anal., 14(4) (1983), 834-844.

[2] G. Bennett, Some elementary inequality III, Quart. J. Math. Oxford Ser., 42 (166) (1991), 149-174.

[3] G. Bennett, Factorizing the classical inequalities, Mem. Amer. Math. Soc., 120 (576) (1996).

[4] S. Bloom and R. Kerman, Weighted norm inequalities for operators of Hardy type, Proc. Amer. Math. Soc., 113(1) (1991), 135-141.

[5] A. Kufner, L. Maligranda and L.-E. Persson, The prehistory of the Hardy inequality, Amer. Math. Monthly, 113 (2006), 715-732.

[6] A. Kufner, L. Maligranda and L.-E. Persson, The Hardy Inequality - About its History and Some Related Results, Vydavetelsky Servis Publishing House, Pilsen, (2007).

[7] A. Kufner and L.-E. Persson, Weighted Inequalities of Hardy Type, World Scientific, New Jersey/London/Singapore/Hong Kong (2003).

[8] C.A. Okpoti, L.-E. Persson and A. Wedestig, Weight characterization for the discrete Hardy inequality with kernel, J. Inequal. Appl., (2006), 1-14.

[9] L. Leindler, Generalization of inequalities of Hardy and Littlewood, Acta Sci. Math. (Szeged), 31 (1970), 279-285. 
